\title{
COMBINED DETAILED AND QUASI STEADY-STATE TIME SIMULATIONS FOR LARGE-DISTURBANCE ANALYSIS
}

\author{
Thierry Van Cutsem \\ FNRS and University of Liège \\ Liège, Belgium \\ t.vancutsem@ulg.ac.be
}

\author{
Marie-Eve Grenier, Daniel Lefebvre \\ Hydro-Québec, TransEnergie division \\ Montréal, Canada \\ lefebvre.daniel.4@hydro.qc.ca
}

\begin{abstract}
This paper deals with the simulation of long-term power system responses to large disturbances in the presence of discrete events. A method combining detailed and quasi steady-state time simulations is presented, the former being used for accuracy and the latter for efficiency reasons. Detailed time simulation is used to analyze the short-term period following a large disturbance and identify the discrete controls triggered. Next, quasi steady-state simulation is used to simulate the same time interval with the discrete controls imposed as external events, before letting the system evolve as usual in the long term. This simple method has been successfully tested on the HydroQuébec system.
\end{abstract}

Keywords - long-term dynamics, time domain simulation, quasi steady-state approximation, voltage stability, frequency dynamics

\section{INTRODUCTION}

$\mathrm{I}^{\mathrm{N}}$ $\mathrm{N}$ power system dynamic studies, the trend is to perform numerical simulations over longer periods of time, with more detailed models, and for more operating conditions and disturbances. However, power system dynamic models are large and involve very different time scales, which makes their simulation over long time intervals very demanding.

To deal with this complexity, variable step size simulation tools have been devised [1]. Nevertheless, many companies use software relying on fixed time step algorithms and do not envisage to change their simulation environment.

An alternative consists in combining detailed and simplified simulation tools [2,3]. The former is used over a time interval following the disturbance, where large transients are caused by the faster dynamics. If the system has survived this period, and once these transients have died out, a simpler model is used in which the faster dynamics are neglected.

The idea of time-scale simplification of a model is not new. It underlies the quasi-sinusoidal (or phasor) approximation used in most stability studies [4], where electromagnetic transients are neglected and the network is modeled by algebraic equations. The idea is further exploited in the Quasi Steady-State (QSS) approximation of long-term dynamics, which consists of replacing the short-term differential equations of generators, motors, compensators, etc. by the corresponding algebraic equilibrium equations [6]. QSS simulation is well suited to computationally intensive tasks such as security limit determination, real-time applications or training simulators $[5,6,7]$.

When combining the detailed and QSS models, however, it is essential to both preserve the reliability of the overall simulation and make the combination of tools totally transparent to the end-user.

A time-scale decomposition-based simulation tool of the type outlined above was already proposed in [2] and has been used for several years by HydroQuébec (H-Q) engineers. Within the context of the $\mathrm{H}-\mathrm{Q}$ migration to another detailed simulation tool, the method has been revisited and a new, easier to implement scheme has been devised.

This paper is organized as follows. Section II states the problem while Section III presents the new approach. Section IV reports on results obtained on the H-Q system. Conclusion and perspectives are offered in Section V.

\section{STATEMENT OF THE PROBLEM}

\section{$2.1 \quad$ System modeling}

In stability studies, the general dynamic model of a power system takes on the form:

$$
\begin{aligned}
\mathbf{0} & =\mathbf{g}\left(\mathbf{x}, \mathbf{y}, \mathbf{z}_{k}\right) \\
\dot{\mathbf{x}} & =\mathbf{f}\left(\mathbf{x}, \mathbf{y}, \mathbf{z}_{k}\right) \\
\mathbf{z}_{k+1} & =\mathbf{h}\left(\mathbf{x}, \mathbf{y}, \mathbf{z}_{k}\right)
\end{aligned}
$$

The algebraic equations (1) relate to the network.

The differential equations (2) relate to a wide variety of phenomena and controls including:

- the short-term dynamics of generators, turbines, governors, Automatic Voltage Regula- 
tors (AVRs), Static Var Compensators (SVCs), induction motors, HVDC links, etc.

- the long-term dynamics of secondary frequency and voltage control, load selfrestoration, etc.

Finally, the discrete-time equations (3) capture discrete events that stem from:

- controllers acting with various delays on shunt compensation, generator setpoints, Load Tap Changers (LTCs), etc.

- equipment protections such as OverExcitation Limiters (OELs), etc.

- system protection schemes against short and long-term instabilities, acting on loads and/or generators.

It must be emphasized that, apart from digital controllers operating at constant sampling rate, the discrete events take place at time instants dictated by the system dynamics itself (which is not captured by the notation).

In the above equations, $\mathbf{y}$ is the vector of bus voltages while $\mathbf{x}$ (resp. $\mathbf{z}_{k}$ ) is the vector of continuous (resp. discrete) states.

In the sequel, the numerical integration of the whole model (1-3) is referred to as Full Time-Scale (FTS) simulation.

\subsection{Principle of the QSS approximation}

As indicated previously, the QSS approximation of long-term dynamics consists of representing faster phenomena by their equilibrium conditions instead of their full dynamics. The correspondingly simplified model takes on the form:

$$
\begin{aligned}
\mathbf{0} & =\mathbf{g}\left(\mathbf{x}_{1}, \mathbf{x}_{2}, \mathbf{y}, \mathbf{z}_{k}\right) \\
\mathbf{0} & =\mathbf{f}_{1}\left(\mathbf{x}_{1}, \mathbf{x}_{2}, \mathbf{y}, \mathbf{z}_{k}\right) \\
\dot{\mathbf{x}}_{\mathbf{2}} & =\mathbf{f}_{2}\left(\mathbf{x}_{1}, \mathbf{x}_{2}, \mathbf{y}, \mathbf{z}_{k}\right) \\
\mathbf{z}_{k+1} & =\mathbf{h}\left(\mathbf{x}_{1}, \mathbf{x}_{2}, \mathbf{y}, \mathbf{z}_{k}\right)
\end{aligned}
$$

in which $\mathbf{x}$ (resp. $\mathbf{f})$ has been decomposed into $\mathbf{x}_{1}$ and $\mathbf{x}_{2}$ (resp. $\mathbf{f}_{1}$ and $\mathbf{f}_{2}$ ).

In long-term voltage stability studies, the shortterm dynamics of generators, turbines and excitation systems can be neglected. Furthermore, (5) may take on the form of three algebraic equations per machine $[5,6]$. The latter account for saturation, AVR voltage droop and governor speed droop effects. Frequency is a component of $\mathbf{x}_{1}$.

If frequency dynamics is of interest, a simplified turbine and governor representation can be retained in (6). Assuming the same speed for all generators and accounting for their inertia, the rate of change of frequency provides an additional equation (6) [8]. Frequency is then a component of $\mathbf{x}_{2}$.

\subsection{Limitation of the QSS approximation}

The QSS approximation is appropriate for checking voltage security with respect to "normal" (typically $\mathrm{N}-1$ ) contingencies [5, 7]. When dealing with severe disturbances, expectedly, the QSS model meets some limitations.

The first limitation lies in the implicit assumption that the neglected short-term dynamics are stable. After a large disturbance, the system may loose stability in the short-term time frame (within - say the first 10 seconds after the disturbance) and hence not enter in the long-term phase simulated under the QSS approximation.

The second limitation is linked to the discrete events represented by (3). A large disturbance may trigger controls with great impact on the system longterm evolution (e.g. shunt compensation switching, underfrequency or undervoltage load shedding, etc.). As already quoted, the sequence of controls depend on the continuous dynamics, and hence may not be correctly identified from the simplified QSS model.

\subsection{Combining detailed and QSS simulations}

The objective of coupling detailed and QSS simulations is to combine the reliability of the former, when dealing with the short-term dynamics, with the efficiency of the latter, when simulating the longterm dynamics.

A first approach was proposed in [2]. In the latter, the detailed model (1-3) is used to analyze the short-term period following a contingency, and once the corresponding dynamics have died out, switching to the QSS model takes place. The state variables of the QSS simulation have to be initialized from the final system state provided by the detailed simulation. Hence, the latter does not start from the steady state provided by a load flow program, as in conventional time simulations, but rather "out of equilibrium". This initialization procedure has to be implemented in the detailed simulation tool, which can be considered as a constraint. Furthermore, the initialization is more delicate when frequency dynamics are included in the QSS model, which was not the case in [2].

The new approach described in the remaining of the paper is free from these drawbacks, since the coupling is performed by post-processing the results of the detailed simulation. 


\section{THE PROPOSED METHOD}

The proposed method consists of the following steps, where the disturbance of concern is applied at $t=0$ and the system response is sought for $t \in\left[0 t_{\text {fin }}\right]$ :

1. run a detailed simulation over the short-term interval $\left[0 t_{s w}\right]$. If the system is unstable, stop;

2. otherwise, identify the discrete events that have occurred over this interval;

3. run a QSS simulation on the same interval, imposing those events as "external disturbances" while preventing the corresponding discrete devices to act by themselves;

4. proceed with the remaining of the QSS simulation, over the $\left.] t_{s w} t_{f i n}\right]$ interval with the automatic devices free to act as usual.

This procedure is justified as follows. Shortly after $t=0$, the short-term dynamics responds to the disturbance with large transients. The full model (1-3) must be used to check system stability and identify the sequence of discrete events. The latter may not be correctly identified from the QSS model (47). However, by imposing the right sequence identified from the detailed model, the QSS system response on $\left[\begin{array}{ll}0 & t_{s w}\end{array}\right]$ is improved and, once the fast transients become small enough, both responses are likely to be close to each other. From there on, the QSS model is a better approximation of the full one and the sequence of discrete controls can be determined on $\left.] t_{s w} t_{f i n}\right]$ with reasonable accuracy.

Let us now illustrate how the discrete events are handled at steps 3 and 4 of the procedure, with a simple logic present in many controllers. The latter consists in comparing a quantity $y$ to a threshold value $y_{\min }$ and taking an action (e.g. switching compensation, shedding load, etc.) if $y<y_{\min }$ for some duration $\tau$.

Consider for instance the situation depicted in Fig. 1. The controller starts its timer at $t=t_{o}$ and should act at $t=t_{1}$, where $t_{1}-t_{o}=\tau$. At step 3 of the procedure, however, the controller is "frozen" and does not act. Instead, the action is imposed at a time $t_{2}$ identified from detailed time simulation (step 2 ). In the shown example, the effect of this action is to bring back $y$ above $y_{\min }$, which stops the timer. Note that if $t_{2}$ was smaller than $t_{1}$, the action would nevertheless be imposed at $t=t_{2}$.

At $t=t_{s w}$, the simulation enters step 4 and the controllers are "freed". Carrying on with the same example, if $y$ falls again below $y_{\min }$ at $t=t_{3}$, the controller acts as usual at $t_{4}=t_{3}+\tau$ since this time is larger than $t_{s w}$.

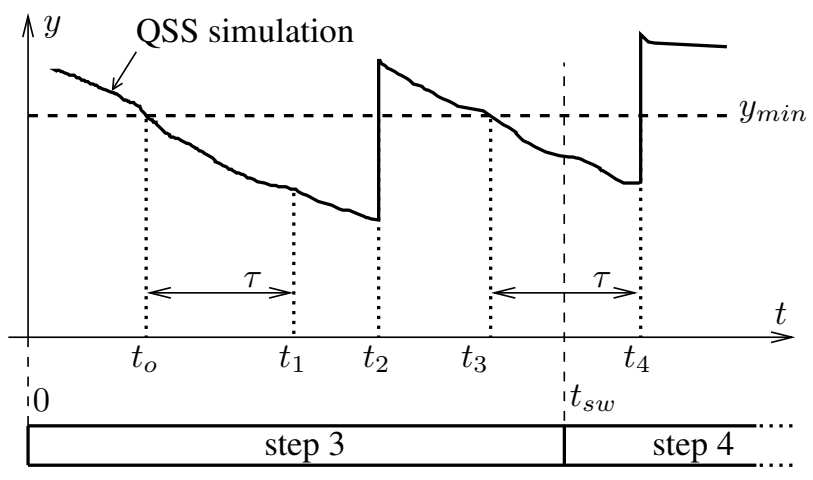

Figure 1: handling of discrete events

As regards the choice of $t_{s w}$, it should be as small as possible to shorten the whole computing time but large enough to guarantee the reliability of the combined simulation. More precisely, it should be large enough to ascertain the short-term stability of the system and correctly identify the discrete events trigerred by the short-term dynamics. This choice is further illustrated in the next section.

\section{RESULTS}

\subsection{The Hydro-Québec system and its model}

With its long $735-\mathrm{kV}$ transmission corridors between the hydro generation areas in the North and the main load centers in the South part of the province, and its isolated mode of operation, the H-Q system is exposed to angle, frequency and voltage stability problems.

Besides static var compensators and synchronous condensers, the automatic shunt reactor switching devices - named MAIS - play an important role in voltage control [9]. These devices, in operation since early 1997, are now available in twenty-two $735-\mathrm{kV}$ substations and control a large part of the total 25,500 Mvar shunt compensation. Each MAIS device relies on the local voltage, the coordination between substations being performed through the switching delays. While fast-acting MAIS can improve transient angle stability, slower MAIS significantly contribute to voltage stability. MAIS devices react to voltage drops but also prevent overvoltages by reconnecting shunt reactors when needed.

Voltage stability is a concern near the load centers of Montréal and Québec city. Long-term voltage stability studies are routinely performed at HydroQuébec using detailed simulation, QSS simulation and the combination of both. The contingencies of concern are the tripping of $735-\mathrm{kV}$ transmission 
lines, especially those feeding the southern part of the system.

The system model includes 846 buses and 132 generators. The discrete events stem from: 371 LTCs acting at different voltage levels with various delays, 89 MAIS devices, 9 OELs protecting the synchronous condensers located near the main load areas, 9 (instantaneous) admittance limiters acting on the SVCs. Fourty-five MAIS react to voltage drops, with thresholds ranging from 0.95 to $0.97 \mathrm{pu}$ and switching delays from 0.7 to 20 seconds.

\subsection{Implementation of the combined simulation}

As regards Step 1 of the proposed method, the simulation stops when no MAIS device has been trigerred over the last 10 seconds of simulated time. This indeed indicates that the short-term dynamics have died out sufficiently, while 10 seconds are enough to detect short-term instability.

As regards the QSS simulation part, the reactor switchings by MAIS devices are discrete events that must be treated as described in Section 3 and Fig. 1, i.e. imposed at Step 3 of the procedure and freed at Step 4. LTCs are treated in the same way, for accuracy reasons. On the other hand, results have shown that OELs and SVC limiters can be left to act as usual during the QSS simulation.

Figure 2 sketches how the coupling is implemented. The ST600 software of H-Q is used for detailed simulation. An interface (hq2ulg) translates the load flow data and extracts the subset of dynamic data relevant to QSS simulation. The latter is performed by the ASTRE software developed at the University of Liège. This procedure has been in use for several years for voltage security assessment against $\mathrm{N}-1$ contingencies [5]. The part shown with dotted lines in Fig. 2 relates to the combined simulation. Namely, ST600 produces a log file with the sequence of discrete events. This ASCII file is read by a small utility (csa) which translates the events into external disturbances to be imposed in the QSS simulation.

Obviously, all these steps are totally transparent to the user. In particular the detailed and QSS simulation plots are assembled as if they were produced by a single tool.

As can be seen, the coupling is simple and can accommodate various detailed simulation softwares, the csa utility being adjusted accordingly. A similar procedure is being devised to couple ASTRE with PTI's PSS/E.

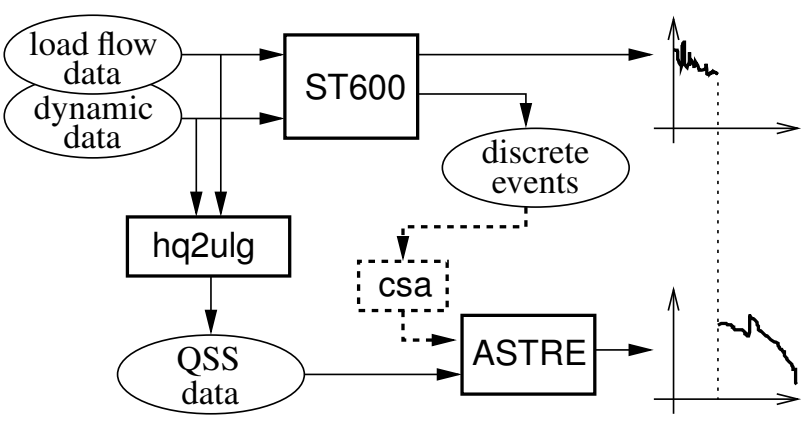

Figure 2: implementation of the combined simulation

\subsection{QSS vs FTS simulation}

The purpose of this section is to illustrate how the QSS model (4-7) approximates the full model (1-3), before reporting on the proposed method.

Figures 3 to 5 deal with the system response to an N-1 contingency, computed under various conditions. The incident is the tripping at $t=1$ of a major $735-\mathrm{kV}$ line of the H-Q system. All the plots of this section show the time evolution of the voltage at the receiving end of the line, located near Montréal. The pre-contingency voltage is $1 \mathrm{pu}$.

The solid line in Fig. 3 relates to the FTS simulation. The latter uses a time step of $0.0083 \mathrm{~s}$ (a halfcycle at $60 \mathrm{~Hz}$ ). Three reactors (of 330-Mvar each) are tripped by MAIS at $t=35.9,93.2$ and 190.4, respectively, as can be seen from the voltage spikes in the figure. The voltage oscillations are caused by the long-term frequency dynamics.

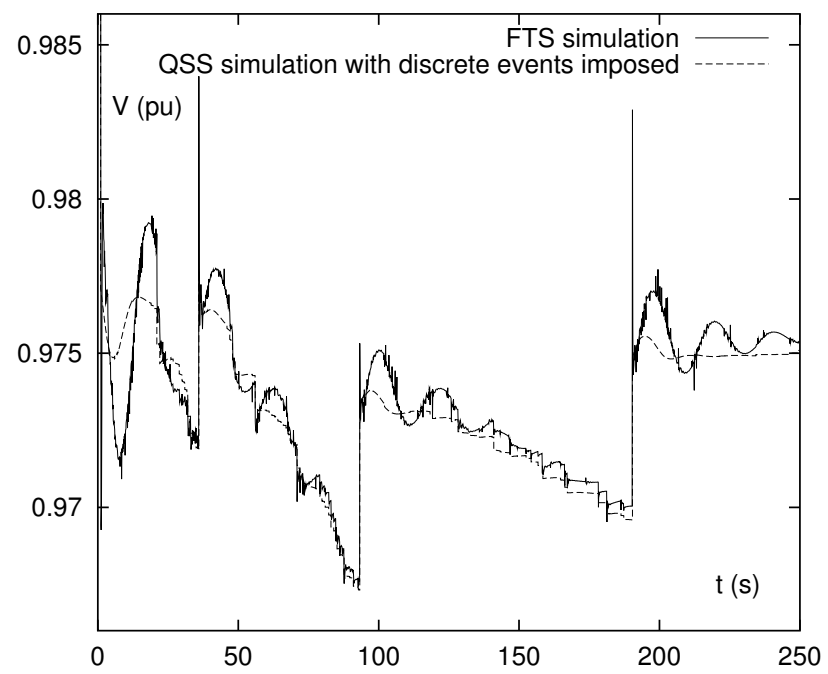

Figure 3: Effect of neglecting short-term dynamics

The dotted line in the same figure relates to a QSS simulation in which all MAIS and LTCs have been frozen for the whole simulation while the corresponding shunt admittances and transformer ratios are forced to change as identified in the FTS simulation. Clearly, there is no gain in computing time to be 
expected from such a simulation (since FTS is used over the whole time interval); the objective is rather to assess the impact of the QSS approximation. Indeed, the difference between the two simulations is only due to the replacement of Eq. (2) by Eqs. $(5,6)$, the discrete changes being the exact ones. As can be seen, the QSS evolution is a very good approximation of the FTS one, although it is 100 to 1000 times faster (as confirmed by the results of Section 4.6).

In Fig. 4, the same FTS simulation is compared to a "traditional" QSS simulation in which the MAIS and LTC changes are decided by the QSS system evolution itself. As can be seen, the two responses differ mainly by the times at which the last two shunt reactors are tripped. This difference is due to shortterm transients. For instance, in the FTS simulation, the voltage spike at $t=35.9$ resets some LTCs (the controlled voltages re-entering the deadbands transiently) and delays their reaction. Since the voltage spike is not present in the QSS response, the LTCs move earlier in the QSS simulation, which causes the voltage to drop and, hence, the second MAIS to be triggered earlier as well.

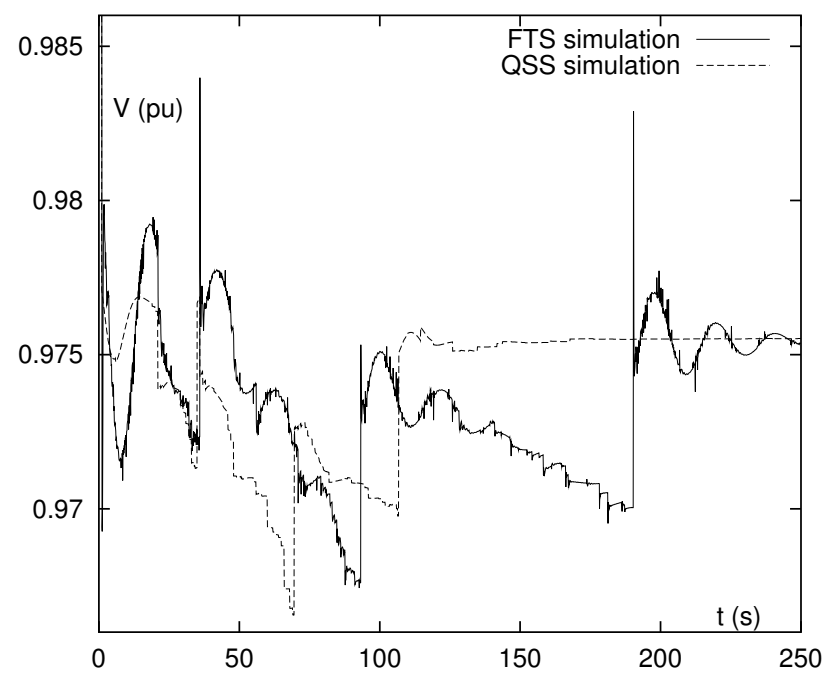

Figure 4: FTS vs QSS simulation

Nevertheless, the QSS output is quite acceptable for this N-1 contingency, since it leads to the right number of shunt reactor trippings and the same final voltage. In fact, the switching times are not considered critical by $\mathrm{H}-\mathrm{Q}$ engineers (even the full model relies on simplifications! These uncertainties are compensated by the closed-loop nature of the MAIS controls). More attention is paid to the number and location of trippings, although a discrepancy by one shunt reactor is still accepted. However, the discrepancy could be larger when the system is subject to a more severe disturbance, which is one motivation for the method presented in this paper.
Figure 5 shows the effect of incorporating frequency dynamics to the QSS model. The QSS evolution with (resp. without) this dynamics is shown with solid (resp. dotted) line and has been computed with a time step of $0.1 \mathrm{~s}$ (resp. $1 \mathrm{~s}$ ). The two curves do not differ very much. The voltage response is a little more accurate when accounting for frequency effects, although this gain does not by itself justify the use of the more refined model, whose computing time is 5 to 10 times longer (although still very short) [8]. Further investigations are needed to improve the QSS model in this respect. Note finally that impedances are updated with frequency in the FTS simulation, while they are kept constant in the QSS one.

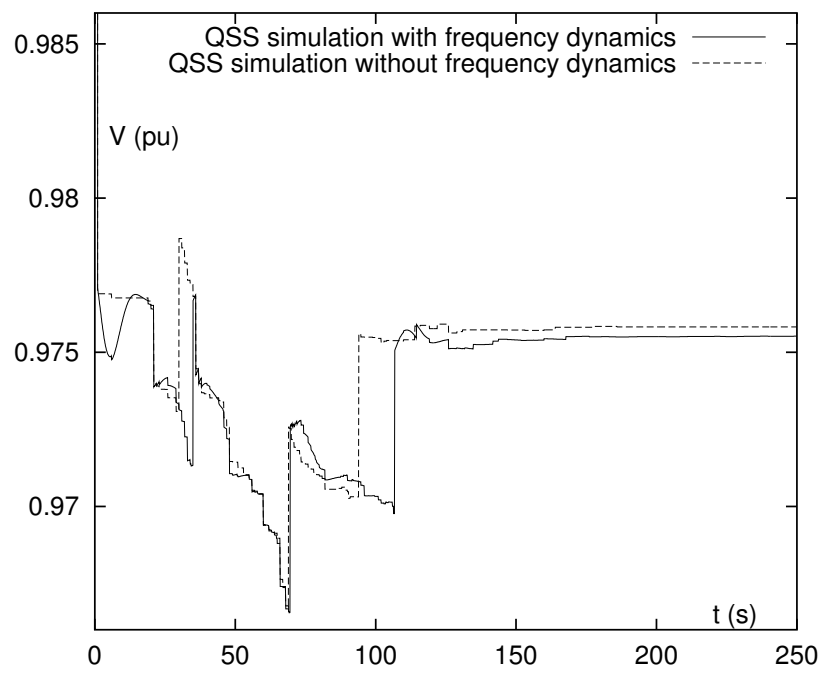

Figure 5: Effect of frequency model in QSS simulation

\subsection{A detailed coupling example}

An example of coupling by the proposed method is given in Fig. 6 where the solid curve relates to the combined simulation and the dotted one to FTS simulation, for comparison purposes. The disturbance of concern is a double line tripping applied at $t=1$.

Using the above mentioned criterion, the detailed simulation stops at $t_{s w}=25$. Over the same $25 \mathrm{sec}-$ onds, a QSS simulation is run with the MAIS and LTC controls frozen, while changes in 6 shunt admittances and 46 transformer ratios are imposed at the various times identified by detailed simulation. The corresponding system evolution is normally not shown to the user, since detailed simulation results are available. This is why a single curve is shown for $t \in\left[\begin{array}{ll}0 & 25\end{array}\right]$ in Fig. 6.

At $t=25$, these controls are released, i.e. they become free to act as usual. The QSS simulation proceeds for $225 \mathrm{~s}$. The corresponding evolution somewhat departs from the FTS reference, for already 
mentioned reasons, but the overall accuracy is good and the system evolution is correctly declared stable.

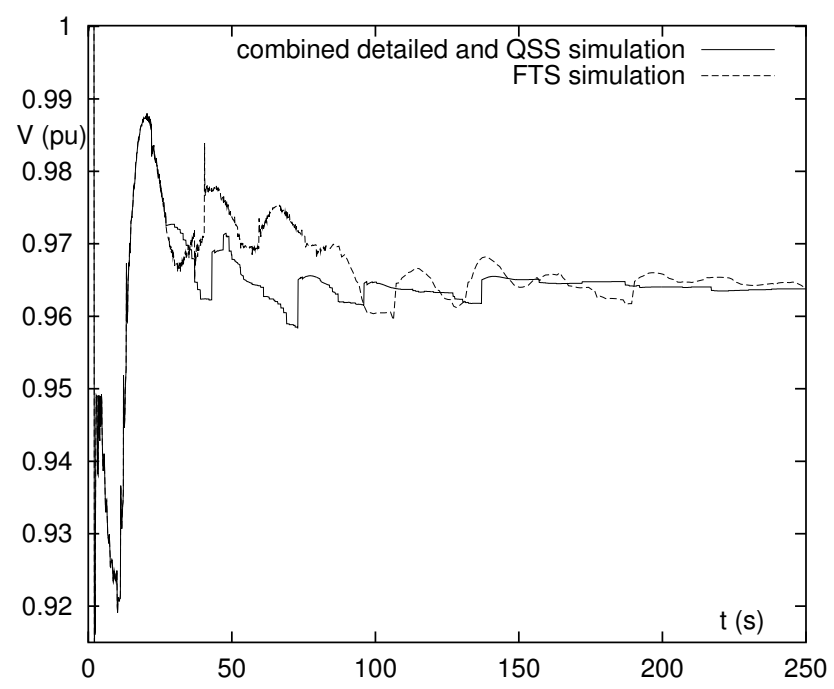

Figure 6: example of coupling

Table 1 details the time and location of shunt reactor trippings in the FTS and combined simulations, respectively. As in the previous example, most switchings take place earlier in the QSS simulation but their number and locations are the same.

\begin{tabular}{|c||c|c||c|c|}
\hline \multicolumn{1}{|c||}{} & \multicolumn{2}{c||}{ FTS } & \multicolumn{2}{c|}{ combined } \\
\hline \multicolumn{1}{|c||}{} & at $t=$ & bus \# & at $t=$ & bus \# \\
\hline \multirow{4}{*}{ step 3 } & 4.0 & 714 & \multicolumn{1}{l|}{} \\
& 11.3 & 715 & \multicolumn{2}{c|}{ same as FTS } \\
& 12.3 & 702 & \multicolumn{2}{c}{} \\
$t \in\left[\begin{array}{ll}0 & 25\end{array}\right]$ & 13.3 & 701 & \multicolumn{2}{c}{} \\
& 14.3 & 707 & & \\
\hline \multirow{3}{*}{ step 4 } & 40.5 & 708 & 43.1 & 708 \\
& 59.4 & 703 & 47.1 & 703 \\
$t \in] 25250]$ & 106.7 & 730 & 77.1 & 730 \\
& 131.9 & 704 & 98.7 & 704 \\
& 189.3 & 713 & 123.3 & 713 \\
\hline
\end{tabular}

Table 1: sequence of shunt trippings

\subsection{Accuracy of security limit determination}

The most appropriate way of checking the accuracy of the proposed method is by computing security margins, which is its main purpose. For a given set of sources and sinks, the secure operation margin is defined as the maximum power transfer increase that still results in a stable post-disturbance evolution [5][7]. A load flow is used to obtain the pre-contingency states and a binary search to determine a stable and an unstable value of the power transfer that differ by less than a tolerance. The latter is set to $100 \mathrm{MW}$.

The margins have been checked on a representative set of 5 scenarios described in Table 2, where the number of switched reactors refers to the marginally stable case.

\begin{tabular}{|c|c|c|c|}
\hline $\begin{array}{c}\text { cont. } \\
\#\end{array}$ & severity & $\begin{array}{c}\text { pre-disturb. } \\
\text { configuration }\end{array}$ & $\begin{array}{c}\text { Nb of switched } \\
\text { reactors }\end{array}$ \\
\hline 1 & $\mathrm{~N}-2$ & intact & 5 \\
2 & $\mathrm{~N}-2$ & 2 lines out & 10 \\
3 & $\mathrm{~N}-2$ & intact & 19 \\
4 & $\mathrm{~N}-2$ & intact & 3 \\
5 & $\mathrm{~N}-1$ & 2 lines out & 3 \\
\hline
\end{tabular}

Table 2: contingency description

For each contingency, Table 3 provides the last stable and the first unstable power increase. The power margins given by the proposed and FTS simulations do not differ by more than $100 \mathrm{MW}$, which is quite accurate for the H-Q system. Furthermore, in terms of tripped reactors, the discrepancy between the proposed and the FTS simulations is zero in almost all cases and never exceeds one, which meets the H-Q criteria.

\begin{tabular}{|c||c|c||c|c|}
\hline \multicolumn{1}{|c||}{} & \multicolumn{2}{c||}{ FTS } & \multicolumn{2}{c|}{ combined } \\
\hline \multicolumn{1}{|c||}{ cont. } & \multicolumn{2}{c||}{ marginally } & \multicolumn{2}{c|}{ marginally } \\
$\#$ & stable & unstable & stable & unstable \\
\hline 1 & 300 & 400 & 400 & 500 \\
2 & 400 & 500 & 400 & 500 \\
3 & 1400 & 1500 & 1400 & 1500 \\
4 & 2400 & 2500 & 2400 & 2500 \\
5 & 1600 & 1700 & 1500 & 1600 \\
\hline
\end{tabular}

Table 3: last stable and first unstable power increases (in MW)

Figures 7 and 8 compare the voltage evolutions provided by the combined and FTS methods in the marginally stable and unstable cases of contingency 2 , respectively. This comparison is demanding since near the stability limit, small changes may later result in large deviations of the system evolution. Nevertheless, the combined simulation reliably fits the FTS one.

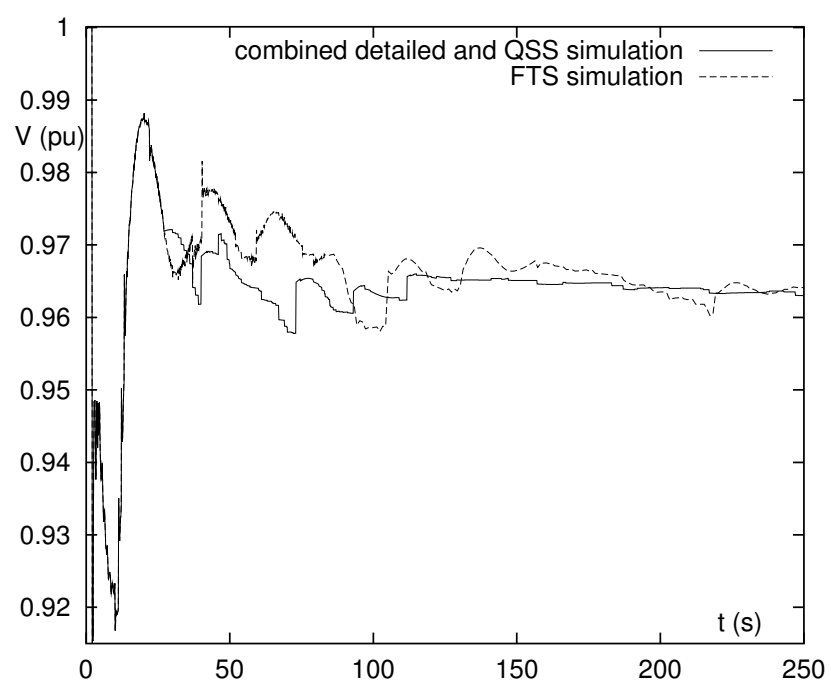

Figure 7: Simulation of marginally stable case 


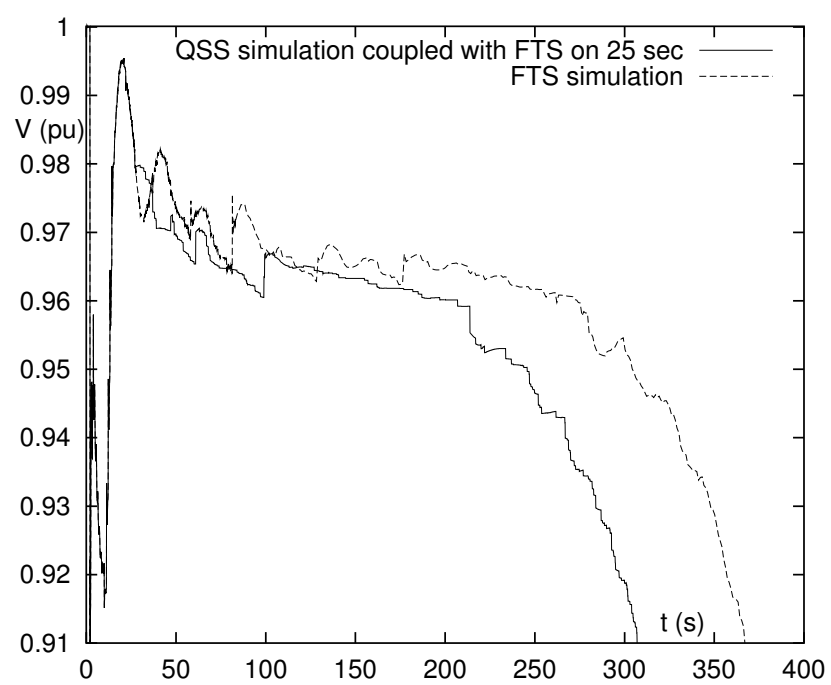

Figure 8: Simulation of marginally unstable case

\subsection{Computational efficiency}

Table 4 gives the computing times of six representative simulations, by the FTS and the proposed methods. For the latter, results are shown as sums of detailed and QSS simulation times. All these times include data reading and have been measured on a 2.2-GHz PC. As can be seen, the proposed method is 4.9 to 8.2 times faster than FTS simulation. These ratios increase to 5.2 and 8.7 if frequency dynamics are not included in the QSS simulation.

\begin{tabular}{|c|c|c||c|c||c|}
\hline$\#$ & $t_{\text {fin }}$ & stable ? & \multicolumn{2}{c||}{ computing times (s) } & gain \\
& $(\mathrm{s})$ & & FTS & combined & \\
\hline 1 & 350 & yes & 893 & $109+10$ & 7.5 \\
2 & 350 & no & 895 & $102+14$ & 7.7 \\
3 & 350 & yes & 954 & $183+10$ & 4.9 \\
4 & 350 & no & 1007 & $171+8$ & 5.6 \\
5 & 300 & yes & 752 & $85+7$ & 8.2 \\
6 & 300 & no & 732 & $86+8$ & 7.8 \\
\hline
\end{tabular}

Table 4: computing times and gain wrt the FTS method

\section{CONCLUSION}

In this paper a new method for the simulation of power system long-term dynamics including discrete events has been presented. It combines the reliability of detailed time simulation with the efficiency of the QSS approximation.

The method for combining the two simulations is simple, while reliable. It is also easier to implement and maintain than the previously used technique, for instance as regards the initialization of the dynamics included in the QSS model. With the proposed scheme, QSS simulation can be coupled to virtually any detailed simulation program, the effort being an adjustment of the procedure to extract the sequence of discrete events from the simulation outputs. The whole procedure can be made transparent to the user, as if a single software was used.

The paper has reported on the good results obtained on the Hydro-Québec system, where the method reveals its ability to account for many discrete events imposed by shunt reactor tripping devices, while reducing the computing time by a factor of 5 to 8 .

\section{ACKNOWLEDGMENT}

Marie-Eve Grenier has taken part in this work in the context of her DEA ("Diplôme d'Etudes Approfondies") degree at the Univ. of Liège. She wants to thank Hydro-Québec for giving her this opportunity.

\section{REFERENCES}

[1] J. Deuse, M. Stubbe, "Dynamic simulation of voltage collapses", IEEE Trans. on Power Systems, Vol. 8, pp. 894-904, 1993

[2] L. Loud, P. Rousseaux, D. Lefebvre, T. Van Cutsem, "A Time-Scale Decomposition-Based Simulation Tool for Voltage Stability Analysis", Proc. IEEE Power Tech conference, Porto (Portugal), 2001, Vol. 2

[3] A. Manzoni, G.N. Taranto, D.M. Falcão, "A comparison of power flow, full and fast dynamic simulations", Proc. 14th Power System Computation Conf., Sevilla, June 2002, session 38

[4] P. Kundur, "Power System Stability and Control", New York, Mc Graw Hill, 1994

[5] T. Van Cutsem, R. Mailhot, "Validation of a fast voltage stability analysis method on the HydroQuébec system", IEEE Trans. on Power Systems, vol. 12, pp. 282-292, 1997

[6] T. Van Cutsem, C. Vournas, Voltage stability of Electric Power systems, Boston, Kluwer Academic Publishers, 1998

[7] C.D. Vournas, G.A. Manos, J. Kabouris, G. Christoforidis, G. Hasse, T. Van Cutsem, "Online voltage Security assessment of the Hellenic Interconnected system", Proc. IEEE Power Tech conf., Bologna, June 2003

[8] M.-E. Grenier, D. Lefebvre, T. Van Cutsem, "A comparison of quasi steady-state models for long-term voltage and/or frequency dynamics simulation", submitted for presentation at IEEE Power Tech conf., St Petersburg, June 2005

[9] S. Bernard, G. Trudel, G. Scott, "A 735-kV shunt reactors automatic switching system for HydroQuébec network", IEEE Trans. on Power Systems, Vol. 11, pp. 2024-2030, 1996 